\title{
Implementation of a phase plate for the generation of homogeneous focal-spot intensity distributions at the high-energy short-pulse laser facility PHELIX
}

\author{
V. Bagnoud ${ }^{1,2}$, J. Hornung ${ }^{1,2}$, M. Afshari ${ }^{1}$, U. Eisenbarth ${ }^{1}$, C. Brabetz ${ }^{1}$, Z. Major ${ }^{1,2}$, and B. Zielbauer ${ }^{1}$ \\ ${ }^{1}$ GSI Helmholtzzentrum für Schwerionenforschung GmbH, Planckstrasse 1, 64291 Darmstadt, Germany \\ ${ }^{2}$ Helmholtz Institute Jena, Fröbelstieg 3, 07743 Jena, Germany \\ (Received 27 August 2019; revised 22 October 2019; accepted 24 October 2019)
}

\begin{abstract}
We propose and demonstrate the use of random phase plates (RPPs) for high-energy sub-picosecond lasers. Contrarily to previous work related to nanosecond lasers, an RPP poses technical challenges with ultrashort-pulse lasers. Here, we implement the RPP near the beginning of the amplifier and image-relay it throughout the laser amplifier. With this, we obtain a uniform intensity distribution in the focus over an area 1600 times the diffraction limit. This method shows no significant drawbacks for the laser and it has been implemented at the PHELIX laser facility where it is now available for users.
\end{abstract}

Keywords: beam shaping; high-power laser; phase plate; short-pulse laser

\section{Introduction}

High-power lasers have proven to be very efficient drivers for plasma physics studies, ever since the invention of lasers in the early 1960s, because of the ability to focus the laser beams to extremely small surfaces, reaching in turn the necessary intensities for laser-plasma interactions ${ }^{[1,2]}$. One of the recurring requirements in laser-plasma interactions is the necessity to create experimental conditions that are nearly one-dimensional, either to drive physical effects or more simply to emulate simulation geometries. Unfortunately, the natural intensity distribution of a laser beam at the focus exhibits strong spatial intensity gradients and is, in nature, fully three-dimensional. In general, quasi-one-dimensional conditions are obtained by the use of random phase plates (RPPs) installed in the beam of the laser, near the end of the laser chain, with the aim to break the spatial coherence of the beam and generate a uniform intensity distribution at the focus ${ }^{[3]}$. In a first approach, the RPP creates a specklelike pattern over a disk that is traditionally near $1 \mathrm{~mm}$ in diameter and the speckle grains have a typical coherence length of a few micrometers. For experiments sensitive to the speckle pattern, additional beam smoothing techniques in one ${ }^{[4]}$ or two ${ }^{[5]}$ dimensions can be applied, resulting in a uniform focal-spot intensity on average.

Correspondence to: V. Bagnoud, GSI Helmholtzzentrum für Schwerionenforschung GmbH, Planckstrasse 1, 64291 Darmstadt, Germany. Email: v.bagnoud@gsi.de
Up to now, the use of RPPs has been restricted to nanosecond high-energy lasers ${ }^{[6,7]}$ with nearly no attempt to employ them with short-pulse high-intensity lasers. There are several reasons for that. First of all, the peak power available with short-pulse lasers was not motivating such development. Indeed, the increase in focus size yields a reduction of the maximum achievable laser intensity. This can be tolerated at nanosecond high-energy lasers that can naturally reach intensities many orders of magnitude above the plasma ionization threshold and can compensate for the intensity reduction. For short-pulse lasers generally aiming at relativistic intensities $\left(I>10^{18} \mathrm{~W} / \mathrm{cm}^{2}\right)$, the peak power necessary to reach a uniform intensity over a modest $100-\mu \mathrm{m}$-diameter disk is around $80 \mathrm{TW}$, a peak power that is now available at many facilities worldwide ${ }^{[8]}$, owing to the rapid development in short-pulse laser technology of the past decade.

A second more obvious reason is the technical complications related to implementing an RPP in a short-pulse laser. In such a case, the standard technique that consists in adding the RPP close to the last focusing optical element cannot be done because the thickness of such an optical element introduces too much deleterious nonlinear Kerr effect. Hence, new strategies for using RPPs with short-pulse high-intensity lasers need to be developed.

In this paper, we discuss the implementation of RPPs with short-pulse lasers, including beam smoothing techniques. In particular, we show and experimentally demonstrate the implementation of a phase plate at the PHELIX laser facility ${ }^{[9]}$ 
Table 1. Maximum RPP substrate thickness for typical short-pulse lasers.

\begin{tabular}{lccc}
\hline Laser type & $\begin{array}{c}\text { Nonlinear index } n_{2} \\
\left(\times 10^{-16} \mathrm{~cm}^{2} / \mathrm{W}\right)\end{array}$ & $\begin{array}{c}\text { Beam max. } \\
\text { fluence }\left(\mathrm{J} / \mathrm{cm}^{2}\right)\end{array}$ & $\begin{array}{c}\text { Max. thickness } \\
(\mu \mathrm{m})\end{array}$ \\
\hline $500 \mathrm{fs}, 1054 \mathrm{~nm}$ & $2.74 \pm 0.17^{[10]}$ & 0.6 & 510 \\
$50 \mathrm{fs}, 800 \mathrm{~nm}$ & $3.0 \pm 0.1^{[11]}$ & 0.3 & 93 \\
\hline
\end{tabular}

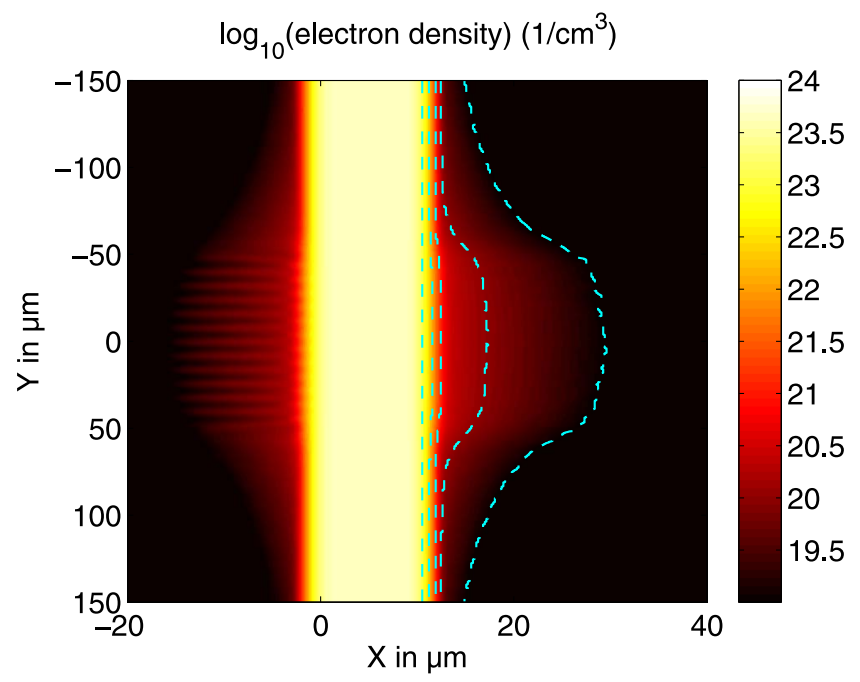

Figure 1. A smooth electron density distribution generated at the back of a target by a periodic illumination pattern of period $10 \mu \mathrm{m}$. The blue dotted lines are equal-density lines at $10^{19}, 10^{20}, 10^{21}, 10^{22}$ and $10^{23}$ electron $/ \mathrm{cm}^{3}$.

to obtain a uniform intensity distribution within a $70 \mu \mathrm{m}$ focal spot, using an F\#1.6 parabola.

\section{Considerations on the use of random phase plates in short-pulse lasers}

Nowadays, the peak power available at laser facilities enables conducting experiments with large laser-intensity distributions of many tens of micrometers in diameter ${ }^{[12]}$. A good strategy to reach this goal is to use a focusing element with a long focal length of up to $30 \mathrm{~m}^{[13]}$, but the result is bulky and limited to a Gaussian intensity distribution at best. Another possibility that is straightforward but very sensitive to the beam quality is to work at an interaction plane outside the laser focus. However, it is well known that large laser systems are prone to beam aberrations that strongly influence the intensity distribution in the intermediate field.

For this reason, the enlargement of the focus with the help of an RPP as used at nanosecond laser facilities comes in question. Beams generated by an RPP are, in general, not 'flat top' but exhibit a speckle pattern of high spatial frequency. This drawback is compensated for by the nanosecond dynamics of the laser-plasma interaction that yields a smoothing of the speckle-pattern effect. For shortpulse lasers, we have verified that such a smoothing effect can be expected in many cases too. For instance, Figure 1 shows the electron density calculated by two-dimensional particle-in-cell simulation at the back of a thin $10 \mu \mathrm{m}$ target illuminated from the left with a $500 \mathrm{fs}$ laser pulse at a peak intensity of $10^{19} \mathrm{~W} / \mathrm{cm}^{2}$ and an illumination pattern defined as a cosine function over a $100 \mu \mathrm{m}$ spot size to emulate the speckle pattern created by the RPP. The electron density does not show any imprint of the high-spatial-frequency laser modulation, which validates our approach.

As stated in Section 1, the difficulty associated with the standard RPPs applied to a short-pulse chirped pulse amplification (CPA) laser is the nonlinear Kerr effect that introduces deleterious spatial and temporal distortions to the pulse when not controlled. For high-energy lasers, B-integral effects yield small-scale focusing and it should be held below 3$5^{[14]}$, while for CPA lasers, spatio-temporal coupling effects command that the B-integral be kept below 1. Assuming that, on the one hand, the RPP is allowed to introduce a B-integral amount of up to 1 and, on the other hand, knowing the maximum average fluence of a beam dictated by the damage threshold of the compression gratings, one can deduce the maximum thickness of the substrate of the RPP for a typical material of low nonlinear refractive index like fused silica. Table 1 gives the results of the estimates for two standard cases: a short 500 fs sub-picosecond laser at $1054 \mathrm{~nm}$ and an ultrashort $50 \mathrm{fs}$ laser at $800 \mathrm{~nm}^{[15]}$, where the maximum tolerable substrate thicknesses are about 500 and $100 \mu \mathrm{m}$, respectively. If the realization of a substrate following the thickness requirements, even at large apertures, might be possible, the manufacturing of a phase plate is a many-step process that requires putting the substrate under mechanical and/or thermal stress. This is not compatible with the substrate thickness requirement and our attempt to find a manufacturer able to work with thin substrates was unfruitful, until now.

A second approach to circumvent the B-integral issue is to write the phase element directly on a reflective optical element (mirror) to be located close to the last focusing element. When considering this option, we faced the problem of the impact of the phase pattern on the damage threshold of the phase mirror and the following optics. Indeed, a decent beam-shaping effect is obtained with a binary phase element, which is cost effective compared to the more advanced technique necessary for continuous-phase-element generation. However, the phase discontinuity locally induces weakness points in the mirror coating. This requires a thorough study of the laser-induced damage mechanisms on the mirror. In addition, strong and uncontrolled intensity modulations appear on the laser beam, already a few centimeters after the phase element, that put the following optics at risk. For this reason, we did not follow this option.

The third possibility is to locate the phase plate where both damage threshold and nonlinear effects are not a problem. This location needs to be before the compressor and, for 


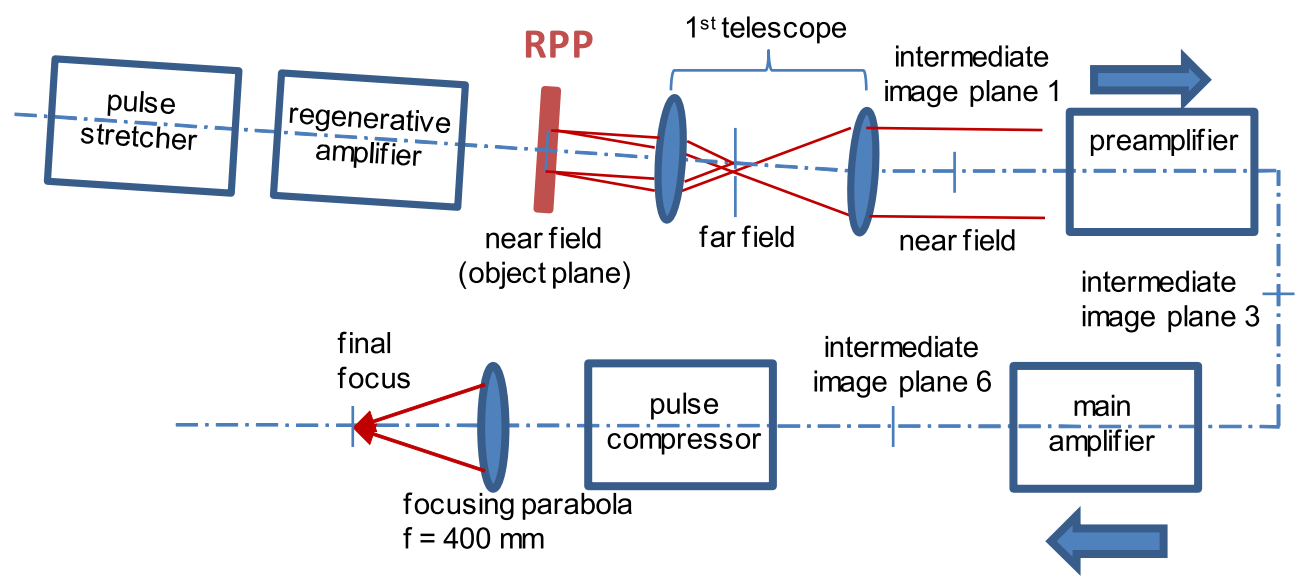

Figure 2. Implementation of the phase plate at the PHELIX facility. The RPP is blazed and operates in the first diffraction order, as schematically shown. A pinhole located in the first telescope removes the zeroth and any other unwanted orders.

obvious economical reasons, we studied the possibility to locate the RPP early in the system, near an image plane, where the beam is still small. This strategy has the advantage to isolate the beam modulation stage from the expensive and damage-threshold-sensitive last optical elements with spatial filters. To do so, the laser system must be adapted to allow for the propagation of a beam that is significantly larger at the far-field location, i.e., in the spatial filters, and exhibits some modulations in the near-field. In addition, the compression of the pulse should not be impacted by the spatial phase of the beam. This condition is fulfilled as long as the spatial gradients in wavefront do not impact the optical path of the single rays in the beam and compression remains homogeneous across the beam in the compressor. This option has been used in the experimental realization at PHELIX and is detailed in the following sections.

\section{Design of a phase plate for the PHELIX facility}

As explained in the previous section, the entrance of the laser pre-amplifier has been chosen for the location of the RPP as shown in Figure 2. PHELIX has a fully imagerelayed amplifier section divided in pre-amplifiers and main amplifiers, where the near-field intensity distribution is defined by a serrated aperture located at the input of the preamplifier and image-relayed by six telescopes throughout the laser, up to the compressor, with a magnification of 33 . Similarly, the far-field intensity distributions are imaged up to the focal plane such that it is easy to design an RPP to replace the serrated aperture and produce the desired far-field intensity distribution. Using PHELIX parameters, a spot of $70 \mu \mathrm{m}$ full width at half maximum (FWHM) at the focus of the $f=400 \mathrm{~mm}$ focusing parabola is desirable. This corresponds to 40 times the diffraction limit in an FWHM sense. In such a case, the RPP should define a beam of $8.47 \times 7.69 \mathrm{~mm}^{2}$ (elliptical) that focuses to a spot of $3.08 \mathrm{~mm}$ (FWHM) at the focus of the first telescope.
One of the aspects of this work is the assessment of the compressor behavior in the case of a spatially modulated beam. Indeed, phase gradients are at the center of concerns because they correspond to a spatially varying change in the local beam-propagation direction $\mathrm{d} \theta$, which in turn locally changes the optical path in the compressor across the beam and induces a change in the compressor dispersion $\mathrm{d} \Phi^{\prime \prime}$. It is, however, possible to anticipate this effect based on a calculation of the simulated wavefront gradients. The horizontal direction is the direction of the highest sensitivity because of the influence of pointing errors on the compression. It is possible to estimate the maximum tolerable gradients by considering the pulse-duration increase for a given local beam-pointing error introduced by the wavefront gradient. Here, we consider the small variation $\mathrm{d} \Phi^{\prime \prime}$ in the second-order term $\left(\Phi^{\prime \prime}=\partial^{2} \Phi / \partial \omega^{2}\left(\omega_{0}\right)\right)$ in the spectralphase expansion and its influence on the pulse duration ${ }^{[16]}$ :

$$
\frac{\tau_{1}}{\tau_{0}}=\sqrt{1+\frac{16 \ln ^{2} 2}{\tau_{0}^{4}} \mathrm{~d} \Phi^{\prime \prime 2}},
$$

where $\tau_{1}$ is the pulse duration resulting from a small increase in group delay dispersion $\mathrm{d} \Phi^{\prime \prime}$ and $\tau_{0}$ the Fourier-transformlimited pulse duration (for a Gaussian pulse, in an FWHM sense). By differentiating the second derivative of the compressor spectral phase $\Phi^{\prime \prime[16]}$ as a function of the incidence angle, we find

$$
\mathrm{d} \Phi^{\prime \prime}=3 \Phi^{\prime \prime} \tan \theta_{d}\left(\omega_{0}\right) \frac{\cos \theta}{\cos \theta_{d}\left(\omega_{0}\right)} \mathrm{d} \theta
$$

with $\theta$ and $\theta_{d}\left(\omega_{0}\right)$ being the incidence angle and the diffracted angle on the compressor grating, respectively. The last two equations can be combined to express $\mathrm{d} \theta$ as a function of the maximum tolerable pulse-duration increase $\tau_{1} / \tau_{0}$. This value is the maximum local deviation $\mathrm{d} \theta$ from the central propagation direction that rays can tolerate. Using the 


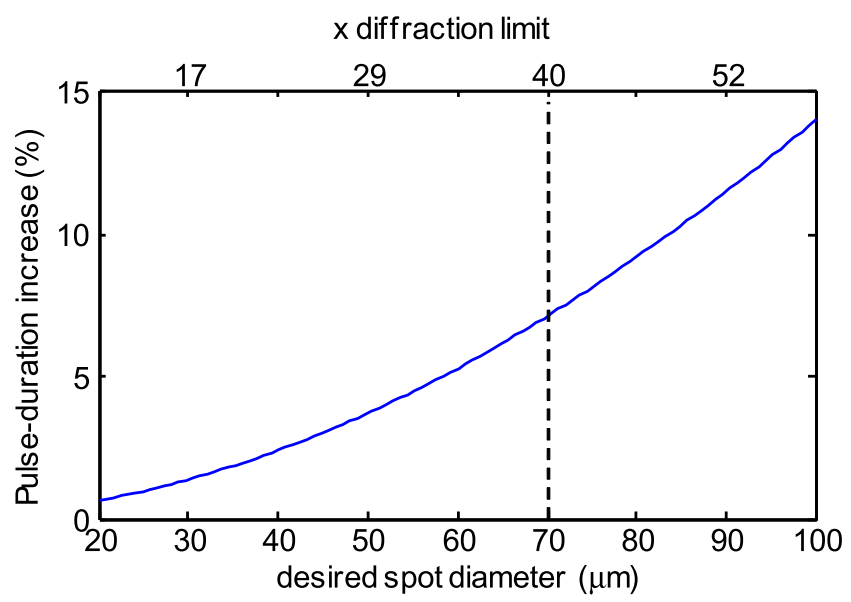

Figure 3. Maximum increase in pulse duration expected as a function of the spot diameter calculated for the PHELIX parameters.

value of the PHELIX compressor $\left(\Phi^{\prime \prime}=1.12 \times 10^{8} \mathrm{fs}^{2}\right)$, an incidence angle of $72.5^{\circ}$ and a pulse duration of $500 \mathrm{fs}$, one can compute the pulse-duration increase as a function of the desired focal-spot diameter $D_{\text {focus }}$ linked to the angle $\mathrm{d} \theta$ by

$$
D_{\text {focus }}<2 f \mathrm{~d} \theta \text {. }
$$

The results are depicted in Figure 3, where the targeted spot size of $70 \mu \mathrm{m}$ yields an increase in the temporal pulse width of $7 \%$. As an additional information, the spot size is also given as a multiple of the diffraction limit, assuming an Airy pattern and an ideal $25-\mathrm{cm}$-large top hat beam. It should be noted that the pulse-duration increase is not homogeneous across the beam focus. In the center and along the vertical direction of the focal spot, the compression is not changed. For most of the applications at our facility, this spatio-temporal coupling feature plays a negligible role, and we decided to validate the design and proceed with the experimental realization.

Another spatio-temporal effect that can impact the pulse shape comes from the compressor configuration found at PHELIX where it is known that a single-pass compressor introduces a pulse front tilt in the far-field ${ }^{[17]}$. However, given the favorable conditions at PHELIX, i.e., a relatively long pulse duration of $500 \mathrm{fs}$ and a large beam-to-stretch ratio of nearly 2 , the effect of the pulse front tilt remains minimal, even for a $70 \mu \mathrm{m}$ focal spot.

We obtained the RPP from a commercial source (SILIOS Technologies) that produces discrete eight-level phase plates inscribed in high-quality substrates with a damage threshold compatible with the operation at a high-power laser facility. The phase mask provides the right beam shaping in the far-field away from the optical axis to avoid a residual peak of intensity in the zeroth order with a high diffraction efficiency. Unfortunately, the phase mask includes spatial discontinuities, i.e., vortices, that translate into speckle-like modulations in the near-field of the beam, as soon as highspatial frequencies are filtered out, which happens already

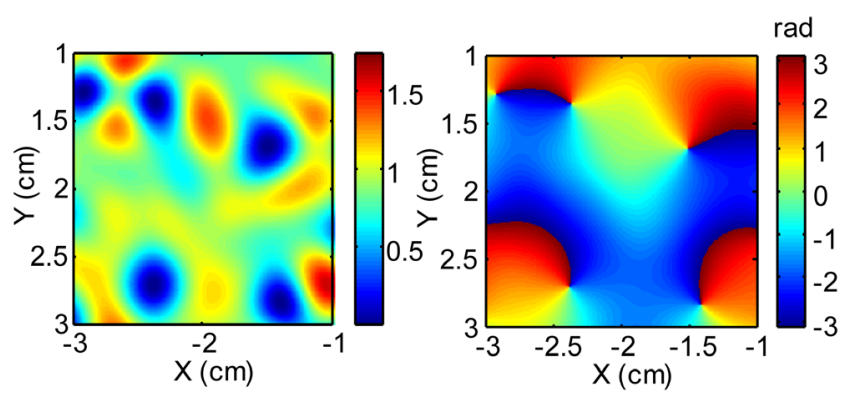

Figure 4. A close-up view of the beam scaled to the main-amplifier dimension. Left: beam intensity normalized to the input beam value; right: phase (radian). The reason for the intensity modulation is the phase discontinuities (e.g., for positions $[-1.5 \mathrm{~cm}, 1.6 \mathrm{~cm}],[-2.4 \mathrm{~cm}, 2.75 \mathrm{~cm}]$ ).

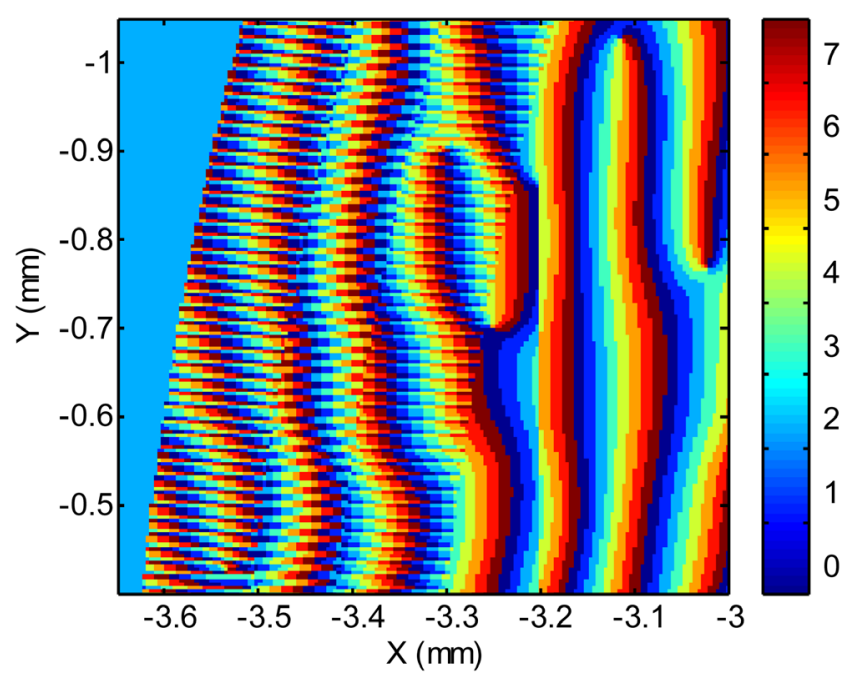

Figure 5. A close-up view of the phase pattern calculated at the edge of the beam to obtain an apodization effect with a discrete eight-level phase object.

in the first spatial filter. This is shown in Figure 4 where the calculated phase (right) and intensity (left) after the first telescope are shown. For the color scale of the intensity plot, a value of 1 corresponds to the intensity of the input beam.

The last feature of the RPP that we employ is a beamshaping function for the near-field. Indeed, it is possible to apply an additional spatial-phase modulation to the mask in order to introduce a smooth intensity shaping in the nearfield ${ }^{[18]}$ and as illustrated in Figure 5. With this feature, the RPP replaces the beam apodizer traditionally used to define the aperture of the laser beam. The RPP has then three distinct areas. Where no transmission is desired, the phase is constant. Here, the incident laser energy is locally not affected by the RPP and propagates up to the zeroth order of the beam in the far-field where it is blocked, hence the very good extinction ratio of the RPP. At the center of the RPP where a $100 \%$ transmission is desired, the standard RPP pattern is applied and, at the edge of the beam, a variable transmission following an error function is applied. In practice, this is done using the following phase amplitude modulation that diffracts the energy away from the first order 
of the RPP:

$$
T(x, y)=\cos ^{2}\left[\frac{\delta \varphi(x, y)}{2}\right]
$$

where $\delta \varphi(x, y)$ is a high-spatial-frequency modulation. Because the RPP is discretized on eight levels, the number of accessible transmission points is also discrete and limited. But to improve this, we increase the number of points by modulating the duty cycle of the phase modulation.

For the beam alignment, switching from the standard serrated aperture to the phase plate requires the use of a pinhole of a different size in the first telescope and its realignment to select the -1 order of the diffracted beam. This is done by re-pointing the input beam. Concomitantly, the filters of the far-field alignment cameras must be changed to accommodate the intensity reduction in the beam throughout the whole amplifier. Note that the main-amplifier spatial filter and other imaging telescopes have large-enough pinholes that work with and without phase plate.

\section{Experimental validation}

The phase plate is made on a square $25 \mathrm{~mm} \mathrm{1-mm-thick}$ fused-silica substrate and is anti-reflection coated. It is inserted in lieu of the standard serrated apodizer in the same motorized holder for transversal centering. The beam has to be tilted to select its first order via the first telescope and a spatial filter (see Figure 2). This is done using the motorized mirrors, located before the RPP, which are used for adjusting the pointing and centering of the input beam. In addition, the pinhole of the first telescope has to be adapted to the size of the far-field that is different from the pinhole required for the beam-shaping function when the serrated apodizer is used.

We conducted a series of shots with and without the RPP. Figure 6 (left) shows the intensity distribution of the beam (near-field) at full energy, where the expected speckle pattern created by the phase discontinuities is clearly visible. The yellow ellipse with a width of $255 \mathrm{~mm}$ and a height of $280 \mathrm{~mm}$ indicates the dimension of the beam, which is slightly elliptical to fit the compressor clear aperture better. The fill factor, defined as the ratio of the beam energy to the energy of an ideal 'top hat' beam of the same surface (the surface of the ellipse equals $561 \mathrm{~cm}^{2}$ ) and maximum peak fluence, equals $25 \%$. Given an allowed operation peak fluence of $0.85 \mathrm{~J} / \mathrm{cm}^{2}$, the highest permitted output energy is reduced to $120 \mathrm{~J}$. This compares to a fill factor of $46 \%$ for the unmodulated beam and a corresponding energy of $224 \mathrm{~J}$. This is best illustrated by the plot on the right-hand side of Figure 6, which shows the histograms of the left beam (green) and of a beam without phase plate (red). For these beams, the ratios of the maximum count to the mean count value are 4 and 2.3, respectively.

The corresponding far-field intensity distribution is presented in Figure 7 that shows the focus of the beam at full
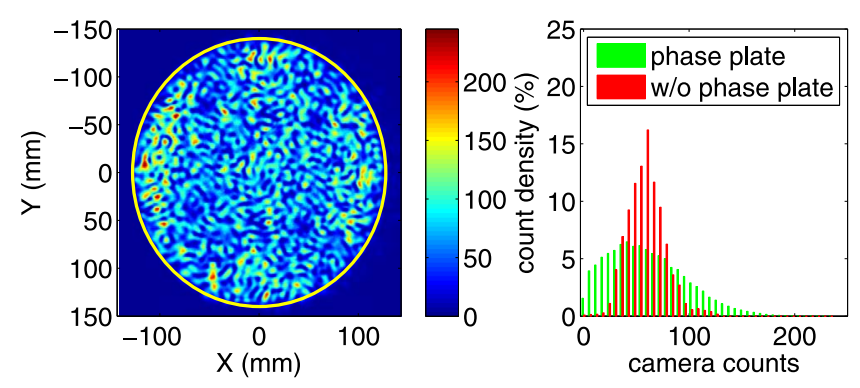

Figure 6. Left: near-field image of the full energy beam. Right: comparison of the image histograms for the modulated beam and a standard beam.

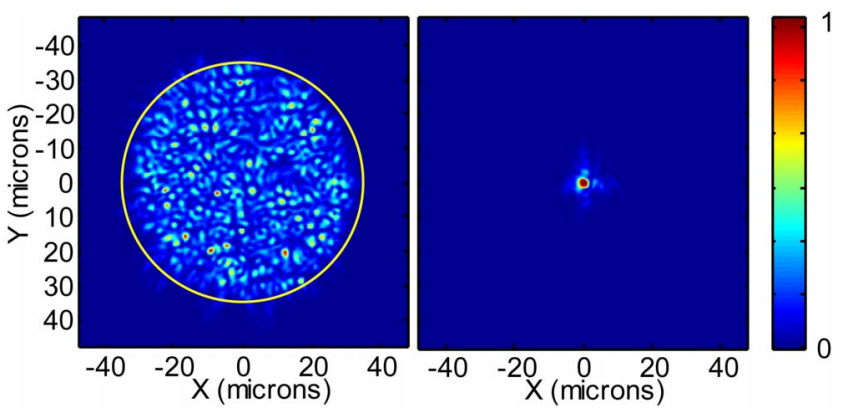

Figure 7. Normalized far-field intensity distributions at the main-amplifier sensor for two shots at $100 \mathrm{~J}$ with (left) and without (right) the RPP. The latter picture is slightly overexposed.

energy (about $100 \mathrm{~J}$ ) during a shot at the end of the main amplifier and recorded at the main-amplifier sensor located at the intermediate image plane 6 (see Figure 2). The image has been scaled to the target plane, whereas the yellow circle delimits the $70 \mu \mathrm{m}$ targeted focal-spot size. The camera has a 16-bit CMOS chip such that the beam attenuation does not need to be changed to compensate for the lower intensity of the beam with RPP (although the standard far-field intensity distribution on the right picture is slightly saturated).

Compared to the standard case, the far-field distribution created when the RPP is used shows a large spot of the expected dimension. In this area, the intensity shows a speckle pattern with a few hot spots. The correlation of the intensity distribution shows a typical speckle grain size of $1.6 \mu \mathrm{m}$, which is consistent with the diffraction limit of the focal spot without phase plate. In addition, a Fourier transform of the intensity distribution shows a slight periodicity in the speckle pattern, which occurs due to the phase object used in the phase plate. However, this periodicity is lost during the propagation of the beam throughout the many amplification stages of the laser system and only shows up in the Fourier analysis of the far-field intensity distribution. Under these conditions, the available average intensity in the $70 \mu \mathrm{m}$ focal spot equals $6 \times 10^{18} \mathrm{~W} / \mathrm{cm}^{2}$ for the maximum allowed energy of $120 \mathrm{~J}$ and assuming a 500 -fs-long pulse. The peak intensity is a factor 6 higher than the average and reaches up to $3.5 \times 10^{19} \mathrm{~W} / \mathrm{cm}^{2}$, which compares to $5 \times 10^{20} \mathrm{~W} / \mathrm{cm}^{2}$, the maximum achievable intensity without phase plate in standard conditions for PHELIX with the same focusing 
parabola. One hypothesis for this larger-than-expected ratio is the on-shot aberration present at PHELIX, whose impact on the speckle distribution remains to be studied thoroughly.

In the temporal domain, the characterization of the laser pulse is not trivial. Indeed, the phase plate creates a nonuniform pulse compression in the far-field. In order to study this, a device capable of spatially resolving the pulse duration across the far-field intensity distribution would be necessary. Our calculation shows that the pulse lengthening with the phase plate used at PHELIX should remain below $10 \%$ at the edge of the far-field distribution, which is relatively small and makes this effect even more complicated to measure. As a rough consistency check, a scanning intensity autocorrelator was used in the diagnostic arm after the compressor to characterize the unamplified beam at $10 \mathrm{~Hz}$. In addition to spatially averaging the above-mentioned spatio-temporal coupling, the intensity autocorrelator setup introduces a blurring effect due to combined interaction angle and nonnegligible spot size of the beam in the doubling crystal (windowing effect), which can be significant with a focal spot about 40 times the diffraction limit and introduces an artificial lengthening of the signal. In the case of the autocorrelator at hand with an internal interaction angle of $15^{\circ}$ and a lens focusing distance of $50 \mathrm{~mm}$, the blurring effect is estimated to exceed the pulse duration itself. In such conditions, no pulse broadening larger than the measurement uncertainty of $50 \%$ was observed.

\section{Conclusion}

We have experimentally investigated the implementation of an RPP at a short-pulse laser facility. For such lasers, the RPP cannot be located at the end of the laser chain because of nonlinear pulse distortions and beam self-focusing. Instead, we have proposed and demonstrated that an RPP located at the beginning of the amplifier is technically feasible and provides a far-field intensity distribution as expected. The main drawback associated with this particular RPP design is a reduction of the maximum allowed energy by nearly a factor of 2 because of phase discontinuities in the near-field. This phase plate has been used in a dedicated beam time where a uniform illumination of a target was necessary for ion and X-ray generation ${ }^{[19]}$. Other applications where onedimensional geometries are needed, like shock physics with ultrashort pulses, will be also tested in the near future.

\section{Acknowledgements}

The authors would like to thank the company SILIOS for the collaborative work in developing the phase plate. This work has been carried out within the framework of the EUROfusion Consortium and has received funding from the European Union's Horizon 2020 research and innovation program under grant agreement number 633053. The views and opinions expressed herein do not necessarily reflect those of the European Commission.

\section{References}

1. P. Mulser, R. Sigel, and S. Witkowski, Phys. Rep. 6, 187 (1973).

2. S. Eliezer, The Interaction of High-Power Lasers with Plasmas (CRC Press, Boca Raton, 2002).

3. Y. Kato, K. Mima, N. Miyanaga, S. Arinaga, Y. Kitagawa, M. Nakatsuka, and C. Yamanaka, Phys. Rev. Lett. 53, 1057 (1984).

4. S. Skupsky, R. Short, T. Kessler, R. Craxton, S. Letzring, and J. Soures, J. Appl. Phys. 66, 3456 (1989).

5. J. E. Rothenberg, Proc. SPIE 2633, 634 (1995).

6. S. Dixit, I. M. Thomas, B. W. Woods, A. Morgan, M. Henesian, P. Wegner, and H. Powell, Appl. Opt. 32, 2543 (1993).

7. G. Collins, L. Da Silva, P. Celliers, D. Gold, M. Foord, R. Wallace, A. Ng, S. Weber, K. Budil, and R. Cauble, Science 281, 1178 (1998).

8. C. N. Danson, C. Haefner, J. Bromage, T. Butcher, J.-C. F. Chanteloup, E. A. Chowdhury, A. Galvanauskas, L. A. Gizzi, J. Hein, D. I. Hillier, N. W. Hopps, Y. Kato, E. A. Khazanov, R. Kodama, G. Korn, R. Li, Y. Li, J. Limpert, J. Ma, C. H. Nam, D. Neely, D. Papadopoulos, R. R. Penman, L. Qian, J. J. Rocca, A. A. Shaykin, C. W. Siders, C. Spindloe, S. Szatmári, R. M. G. M. Trines, J. Zhu, P. Zhu, and J. D. Zuegel, High Power Laser Sci. Eng. 7, e54 (2019).

9. V. Bagnoud, B. Aurand, A. Blazevic, S. Borneis, C. Bruske, B. Ecker, U. Eisenbarth, J. Fils, A. Frank, E. Gaul, S. Goette, C. Haefner, T. Hahn, K. Harres, H.-M. Heuck, D. Hochhaus, D. H. H. Homann, D. Javorková, H.-J. Kluge, T. Kuehl, S. Kunzer, M. Kreutz, T. Merz-Mantwill, P. Neumayer, E. Onkels, D. Reemts, O. Rosmej, M. Roth, T. Stoehlker, A. Tauschwitz, B. Zielbauer, d. Zimmer, and K. Witte, Appl. Phys. B 100, 137 (2010).

10. D. Milam, Appl. Opt. 37, 546 (1998).

11. C. Karras, D. Litzkendorf, S. Grimm, K. Schuster, W. Paa, and H. Stafast, Opt. Mater. Express 4, 2066 (2014).

12. A. Gonsalves, K. Nakamura, J. Daniels, C. Benedetti, C. Pieronek, T. de Raadt, S. Steinke, J. Bin, S. Bulanov, J. van Tilborg, C. G. R. Geddes, C. B. Schroeder, C. Tóth, E. Esarey, K. Swanson, L. Fan-Chiang, G. Bagdasarov, N. Bobrova, V. Gasilov, G. Korn, P. Sasorov, and W. P. Leemans, Phys. Rev. Lett. 122, 084801 (2019).

13. J. Zou, C. Le Blanc, D. Papadopoulos, G. Chériaux, P. Georges, G. Mennerat, F. Druon, L. Lecherbourg, A. Pellegrina, P. Ramirez, F. Giambruno, A. Fréneaux, F. Leconte, D. Badarau, J. M. Boudenne, D. Fournet, T. valloton, J.-L. Paillard, J. L. Veray, M. Pina, P. Monot, J.-P. Chambaret, P. Martin, F. Mathieu, P. Audebert, and F. Amirano, High Power Laser Sci. Eng. 3, e2 (2015).

14. A. E. Siegman, Lasers (University Science Books, Mill Valley, 1986), p. 385

15. T. J. Yu, S. K. Lee, J. H. Sung, J. W. Yoon, T. M. Jeong, and J. Lee, Opt. Express 20, 10807 (2012).

16. F. Träger, Springer Handbook of Lasers and Optics (Springer Science \& Business Media, 2007), p. 945.

17. G. Pretzler, A. Kasper, and K. Witte, Appl. Phys. B 70, 1 (2000).

18. V. Bagnoud and J. D. Zuegel, Opt. Lett. 29, 295 (2004).

19. M. Afshari, J. Hornung, A. Kleinschmidt, P. Neumayer, D. Bertini, and V. Bagnoud, Phys. Plasmas, submitted. 